\title{
Frequency of nodular goiter and autoimmune thyroid disease and association of these disorders with insulin resistance in polycystic ovary syndrome
}

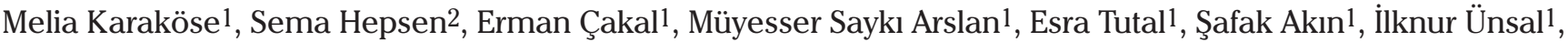 \\ Mustafa Özbek 1 \\ 1Department of Endocrinology and Metabolism, Dışkapı Training and Research Hospital, Ankara, Turkey \\ 2Department of Internal Medicine, Dışkapı Training and Research Hospital, Ankara, Turkey
}

\section{Abstract}

Objective: Polycystic ovary syndrome (PCOS) is a frequent endocrine disease in women. Nodular goiter and autoimmune thyroid disease (AITD) are endocrinologic abnormalities that have high prevalence. The purpose of our study was to detect the prevalence of AITD and nodular goiter in patients with PCOS and investigate whether PCOS-related hormones and metabolic factors affect these thyroid disorders.

Material and Methods: Ninety-seven women with PCOS and 71 healthy female volunteers were recruited into the study. Serum-free thyroxine, thyroid-stimulating hormone, anti-thyroperoxidase antibody and anti-thyroglobulin antibody levels were evaluated. Thyroid volume (TV) was calculated using ultrasonography.

Results: The body mass index (BMI), Waist/Hip ratio, homeostasis model assessment insulin resistance (HOMA-IR), fasting blood glucose, triglyceride and low-density lipoproteins, and fasting insulin were significantly higher in the PCOS group ( $<<.05)$. The control group had significantly higher serum high density lipoprotein cholesterol results $(\mathrm{p}=.005)$. The mean TV was $11.4 \pm 4.7 \mathrm{~mL}$ in the PCOS group and $9.9 \pm 2.8$ $\mathrm{mL}$ in the controls $(\mathrm{p}=.022)$. Twenty-nine patients with PCOS $(29 / 97 ; 29.9 \%)$ had thyroid nodules, whereas only eleven control subjects had thyroid nodules $(11 / 71 ; 15.5 \%)(p=.043)$. The frequency of AITD was significantly higher in the PCOS group $(p=.001)$. A statistically significant relationship was found between TV and age, fasting glucose, HOMA-IR, BMI, and fasting insulin ( $\mathrm{p}<.05)$. Participants with thyroid nodules were older and had higher fasting glucose, BMI, fasting insulin, and HOMA-IR values compared with those without thyroid nodules ( $\mathrm{p}<.05$ ).

Conclusion: We demonstrated that TV and frequency of nodular goiter were increased in patients with PCOS. This result was related with insulin resistance. Therefore, we recommend that patients with PCOS must be investigated for the development of nodular goiter and AITD. (J Turk Ger Gynecol Assoc 2017; 18: 85-9)

Keywords: Polycystic ovary syndrome, autoimmune thyroid disease, nodular goiter, insulin resistance

Received: 13 December, 2016 Accepted: 2 March, 2017

\section{Introduction}

Polycystic ovary syndrome (PCOS) is a frequent endocrine disease with a prevalence of $5-10 \%$ in women at reproductive age (1). Hyperandrogenism, menstrual irregularities, infertility, and obesity are main features of this syndrome. Patients with PCOS have increased risk of metabolic syndrome, cardiovascular disease, insulin resistance, type 2 diabetes mellitus (type 2 DM), and endometrial carcinoma (2-6).

Nodular goiter and autoimmune thyroid disease (AITD) are both frequently seen disorders in endocrine practice (7). Autopsy and ultrasonography (USG) series showed that thyroid nodules had a high prevalence (19-50\%) in the general population (8, 9). In women, the most common cause of hypothyroidism is AITD, which affects 5-20\% of the young female population. The interaction of genetic and hormonal factors are important in the etiology of AITD $(10,11)$.

Although iodine deficiency, female sex and age are well-known risk factors for nodular goiter, the exact pathogenesis remains to be illuminated. The potential relation between insulin resistance and thyroid nodules was reported by Rezzonico et al. $(12,13)$, and insulin resistance was also accepted as a 
risk factor for cancer development. Patients with PCOS have increased risk of insulin resistance. The purpose of this study was to determine the prevalence of AITD and nodular goiter in patients with PCOS and investigate whether there was an effect of PCOS-related hormones and metabolic factors on these thyroid disorders.

\section{Material and Methods}

Ninety-seven patients who were diagnosed as having PCOS between August 2015 and September 2016 in our hospital at the Department of Endocrinology and Metabolism outpatient clinic in accordance with the 2003 Rotterdam criteria were recruited to the study (14). Seventy-one age-matched healthy female volunteers were recruited to the study as a control group. The exclusion criteria were chronic systemic disease, and using drugs that could affect insulin sensitivity and lipid parameters. The study protocol was approved by the Ethics Department and all participants gave informed consent.

Anthropometric measurement, physical examination, and biochemical screening were made in all patients and controls. We obtained fasting blood samples from all participants during the $2^{\text {nd }}-5^{\text {th }}$ days of the menstrual cycle. Hormonal and metabolic variables of all participants were assessed. Insulin resistance was calculated using the homoeostasis model assessment formula (15). Body mass index (BMI) and Waist/ Hip ratio (WHR) were determined.

Chemiluminescent microparticle immunoassays (Abbott, Architect i2000, Abbott Laboratories Diagnostics Division, IL, USA) were used to measure thyroid-stimulating hormone (TSH) and free thyroxine $\left(\mathrm{fT}_{4}\right)$, free triiodothyronine $\left(\mathrm{fT}_{3}\right)$. Chemiluminescent competitive immunoassays (Advia centaur XP, Siemens, Tarrytown, USA) were used to measure the antithyroglobulin antibody (anti-Tg $\mathrm{Ab}$ ) and anti-thyroperoxidase antibody (anti-TPO Ab). The lower and upper limits were as follows: $\mathrm{fT}_{3}$ : $2.5-3.9 \mathrm{pg} / \mathrm{mL} \mathrm{fT}_{4}: 0.58-1.60 \mathrm{ng} / \mathrm{dL}$; TSH: $0.38-5.33$ $\mu \mathrm{IU} / \mathrm{mL}$; anti-Tg Ab: 0-60 IU/mL; anti-TPO Ab:0-57 IU/mL.

Thyroid USG was performed with a high-resolution ultrasound machine (Hitachi, Japan; EUB 7000) that had a 6-14 megahertz linear transducer. Only one operator performed all the measurements. Lesions over $3 \mathrm{~mm}$ (diameter) on USG were considered as nodules. The elliptical shape volume formula (0.479 $\mathrm{x}$ length $\mathrm{x}$ width $\mathrm{x}$ height) was used to calculate the volume of each thyroid lobe, and total thyroid volume (TV) was calculated by adding the right and left lobe volumes.

\section{Statistical analysis}

The SPSS statistical software (version 17; SPSS, Chicago, IL, USA) was used to perform the statistical analysis. Fisher's exact test or Chi-square test were used to analyze categorical variables. Normality of the variables was tested using the KolmogorovSmirnov test. The Mann-Whitney $U$ test and independent samples t-test were to compare groups. Data are expressed as mean \pm standard deviation or median with interquartile range as appropriate. Continuous variables were evaluated using Pearson's correlation coefficient, and Spearman's rho correlation coefficient test was used to evaluate non-normally distributed variables. $\mathrm{P}$ values less than 0.05 were considered statistically significant.

\section{Results}

The study included 97 patients with PCOS (mean age, 24.1 \pm 6.0 years) and 71 controls (mean age, 24.4 4.5 years). Table 1 represents the general characteristics of the patients and controls. There were no significant differences in terms of age and total cholesterol levels between the groups ( $p>.05)$. The BMI, WHR, triglyceride (TG), low density lipoprotein cholesterol,

Table 1. The clinical and biochemical data of patients with polycystic ovary syndrome and controls

\begin{tabular}{|c|c|c|c|}
\hline Variable & $\operatorname{PCOS}(n=97)$ & Controls $(n=71)$ & $\mathbf{p}$ \\
\hline Age, years & $24.1 \pm 6.0$ & $24.4 \pm 4.5$ & .759 \\
\hline BMI, $\mathrm{kg} / \mathrm{m}^{2}$ & $27.5 \pm 6.0$ & $23.4 \pm 5.0$ & $<.001$ \\
\hline Waist/Hip ratio & $0.84 \pm 0.09$ & $0.75 \pm 0.06$ & $<.001$ \\
\hline Fasting glucose, mg/dL & $85.6 \pm 8.9$ & $80.2 \pm 8.9$ & $<.001$ \\
\hline Fasting insulin, $\mu$-IU/mL & $15.8 \pm 7.1$ & $11.4 \pm 5.2$ & $<.001$ \\
\hline HOMA-IR & $3.4 \pm 1.7$ & $2.2 \pm 1.2$ & $<.001$ \\
\hline Total cholesterol, mg/dL & $177.9 \pm 30.6$ & $169.3 \pm 26.1$ & .060 \\
\hline Triglyceride, $\mathrm{mg} / \mathrm{dL}$ & $112.5 \pm 64.3$ & $89.6 \pm 44.5$ & .023 \\
\hline HDL-C, mg/dL & $53.1 \pm 13.3$ & $59.5 \pm 15.1$ & .005 \\
\hline LDL-C, mg/dL & $104.6 \pm 24.0$ & $87.4 \pm 18.2$ & $<.001$ \\
\hline
\end{tabular}


fasting blood glucose, fasting insulin and homeostasis model assessment insulin resistance (HOMA-IR) were significantly higher in patients with PCOS than in the control group $(\mathrm{p}<0.001, \mathrm{p}<0.001, \mathrm{p}=0.023, \mathrm{p}<0.001, \mathrm{p}<0.001, \mathrm{p}<0.001$, $\mathrm{p}<0.001$, respectively). High density lipoprotein cholesterol was significantly higher in the control group $(\mathrm{p}=0.005)$.

The $\mathrm{TSH}$ and $\mathrm{fT}_{4}$ levels were similar in both groups. Table 2 represents the thyroid examination of the patients and controls. The mean TV was $11.4 \pm 4.7 \mathrm{~mL}$ in patients with PCOS and $9.9 \pm 2.8 \mathrm{~mL}$ in healthy controls $(\mathrm{p}=.022)$. In the PCOS group and controls, thyroid nodules were detected in 29/97 (29.9\%) and $11 / 71(15.5 \%)$, respectively $(p=.043)$. The number of patients with PCOS who had a single nodule was 23 and multiple nodules was 6 . In contrast, 6 control subjects had a single nodule and 5 had multiple nodules.

Patients with PCOS had higher a prevalence of positive anti$\mathrm{Tg}$ Ab than controls ( $16.5 \%$ vs. $5.6 \%)(\mathrm{p}=.051)$. The prevalence of subjects with positive anti-TPO Ab among the patient and control groups was $32.0 \%$ and $15.5 \%$, respectively. It was significantly higher in patients with PCOS $(p=.019)$. The frequency of AITD was significantly higher in patients with PCOS [PCOS, 39/97 (40.2\%); and controls, 11/71 (15.5\%); $\mathrm{p}=.001]$ when we consider either thyroid heterogeneity and/or positivity of autoantibodies as AITD.

There were statistically significant relationships between TV and age, BMI, fasting glucose, HOMA-IR, and fasting insulin (Table 3, Figure 1). The correlation between anti-TG Ab, anti-TPO Ab and TSH levels were also statistically significant $(\mathrm{r}=0.466, \mathrm{p}<.001 ; \mathrm{r}=0.218, \mathrm{p}=.005$, respectively).

The participants were divided into two groups according to the existence of thyroid nodules. Participants with thyroid nodules were older and had higher fasting glucose, fasting insulin, HOMA-IR, and BMI compared with those without thyroid nodules (Table 4). $\mathrm{TSH}_{\text {, }} \mathrm{fT}_{4}$, anti-Tg $\mathrm{Ab}$, anti-TPO Ab, and total TV were similar both groups.

Table 2. Thyroid test results of the patients with polycystic ovary syndrome and controls

\begin{tabular}{|c|c|c|c|}
\hline Variable & $\operatorname{PCOS}(n=97)$ & Controls $(n=71)$ & $\mathbf{p}$ \\
\hline $\mathrm{TV}, \mathrm{mL}$ & $11.4 \pm 4.7$ & $9.9 \pm 2.8$ & .022 \\
\hline Thyroid nodule & $29(29.9 \%)$ & $11(15.5 \%)$ & .043 \\
\hline Anti-Tg Ab positive & $16(16.5 \%)$ & $4(5.6 \%)$ & .051 \\
\hline Anti-Tg Ab negative & $81(83.5 \%)$ & $67(94.4 \%)$ & \\
\hline Anti-TPO Ab positive & $31(32 \%)$ & $11(15.5 \%)$ & .019 \\
\hline Anti-TPO Ab negative & $66(68 \%)$ & $60(84.5 \%)$ & \\
\hline AITD & $39(40.2 \%)$ & $11(15.5 \%)$ & .001 \\
\hline $\mathrm{TSH}, \mu \mathrm{IU} / \mathrm{mL}$ & $2.4 \pm 1.2$ & $2.0 \pm 1.0$ & .243 \\
\hline $\mathrm{fT}_{4}, \mathrm{ng} / \mathrm{dL}$ & $1.1 \pm 0.2$ & $1.1 \pm 0.1$ & .905 \\
\hline
\end{tabular}
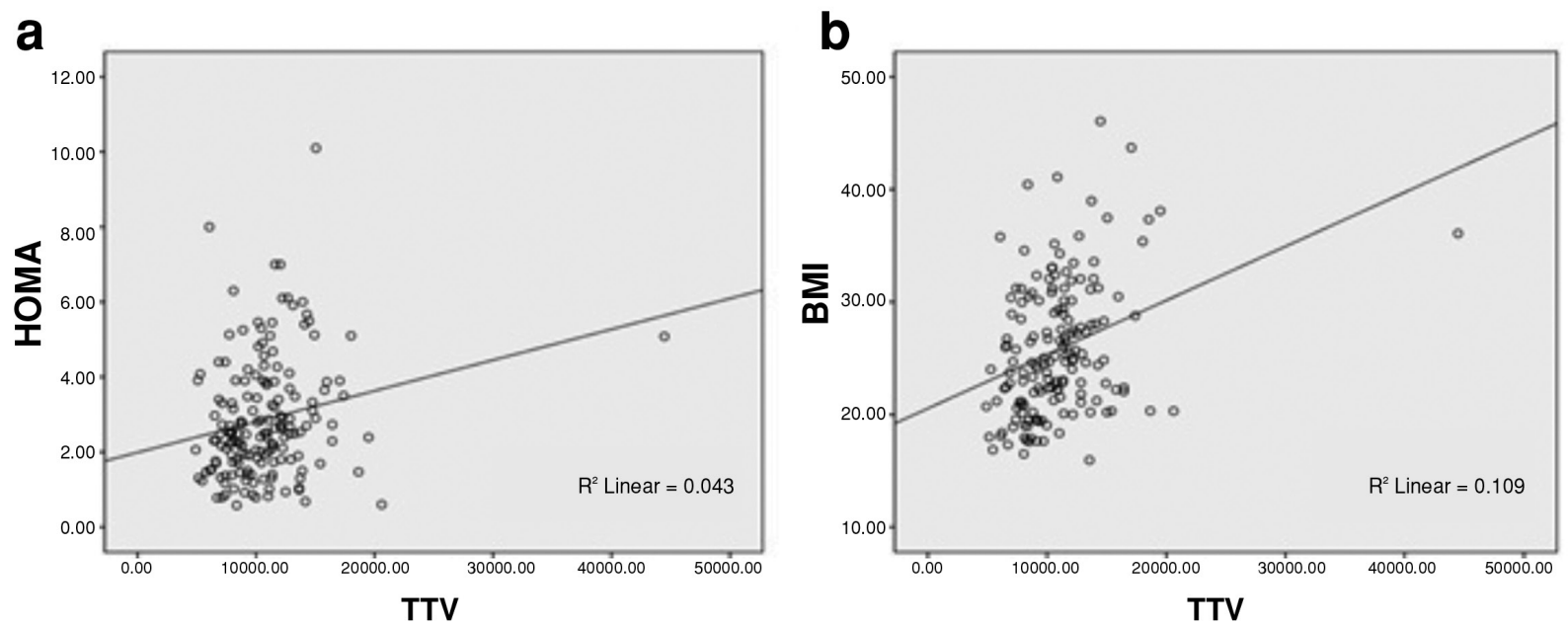

Figure 1. Thyroid volume was correlated with (a) homeostasis model assessment insulin resistance and (b) body mass index 
Table 3. The correlation of thyroid volume with other parameters

\begin{tabular}{|l|l|l|}
\hline Variable & $\mathbf{~}$ & $\mathbf{p}$ \\
\hline Age (years) & 0.172 & $\mathbf{. 0 2 6}$ \\
\hline BMI $\left(\mathrm{kg} / \mathrm{m}^{2}\right)$ & 0.330 & $<.001$ \\
\hline Fasting glucose & 0.195 & $\mathbf{. 0 1 2}$ \\
\hline Fasting insulin & 0.212 & $\mathbf{. 0 0 6}$ \\
\hline HOMA-IR & 0.208 & $\mathbf{. 0 0 7}$ \\
\hline $\begin{array}{l}\text { BMI: body mass index; HOMA-IR: homeostasis model assessment insulin } \\
\text { resistance index } \\
\text { r, Pearson's correlation coefficient }\end{array}$ \\
\hline
\end{tabular}

Table 4. Comparison of clinical and biochemical data in participants with and without thyroid nodules

\begin{tabular}{|l|l|l|l|}
\hline Variable & $\begin{array}{l}\text { Thyroid } \\
\text { nodule } \\
(+)\end{array}$ & $\begin{array}{l}\text { Thyroid } \\
\text { nodule } \\
(-)\end{array}$ & p \\
\hline Age, years & $27.0 \pm 6.4$ & $23.4 \pm 4.8$ & $\mathbf{. 0 0 1}$ \\
\hline BMI, kg/m ${ }^{2}$ & $29.1 \pm 6.4$ & $24.8 \pm 5.4$ & $<.001$ \\
\hline Fasting glucose, $\mathrm{mg} / \mathrm{dL}$ & $87.0 \pm 10.8$ & $82.2 \pm 8.4$ & $\mathbf{. 0 0 5}$ \\
\hline Fasting insulin, $\mu$-IU/mL & $16.6 \pm 6.4$ & $13.1 \pm 6.6$ & $\mathbf{. 0 0 4}$ \\
\hline HOMA-IR & $3.5 \pm 1.6$ & $2.7 \pm 1.5$ & $\mathbf{. 0 0 2}$ \\
\hline $\begin{array}{l}\text { BMI: body mass index; HOMA-IR: homeostasis model assessment insulin } \\
\text { resistance index } \\
\text { Values are shown as mean } \pm \text { standard deviation }\end{array}$ \\
\hline
\end{tabular}

\section{Discussion}

Our study showed that nodule frequency and TV were significantly higher in patients with PCOS. A positive correlation was detected between TV and age, BMI, fasting glucose, fasting insulin, and HOMA-IR. Additionally, participants with thyroid nodules were older and had higher BMI, fasting glucose, fasting insulin, and HOMA-IR compared with those without thyroid nodules.

Duran et al. (16) investigated the nodular goiter prevalence in patients with PCOS. They reported that patients and controls had similar TV and nodule frequency. In their study, age and TV had a positive correlation. However, two different studies demonstrated that thyroid disorders had a high prevalence rate among young patients with PCOS compared with age-matched controls $(17,18)$.

TSH has important roles in the differentiation and growth of thyroid cells (19). In our study, serum TSH levels were normal and not different between patients and controls. In addition, TSH levels were similar between participants with- and without thyroid nodules. Therefore, TSH cannot be the only factor in the pathogenesis of nodule formation. Rezzonico et al. (12) reported the mitogenic effect of insulin on thyroid cell cultures.
Other studies confirmed the relationship between thyroid nodule and insulin resistance (20). The reduction of thyroid nodule volume after amelioration of insulin resistance with metformin proved this causal relationship (21).

Patients with PCOS frequently have hyperinsulinemia and insulin resistance (14). We detected higher fasting insulin and HOMAIR values in patients with thyroid nodules. The characteristic features of insulin resistance are hyperinsulinemia and impaired biologic response to insulin within target tissues (22, 23). It has been also shown that thyroid cancer cells have insulin receptors $(24,25)$. In light of our study results, we recommend USG evaluation for the presence of thyroid nodules in patients with PCOS with insulin resistance.

The association between AITD with PCOS is an another important topic. Kachuei et al. (26) and Janssen et al. (27) reported that the prevalence of autoimmune thyroiditis in PCOS was high $(28,29)$. We also detected statistically significantly high levels of anti-Tg and anti-TPO in patients with PCOS. The prevalence of positive anti-TPO $\mathrm{Ab}$ and anti-TgAb was higher in the PCOS group than in controls. Also the AITD's frequency was significantly higher in patients with PCOS. Calvar et al. (28) demonstrated that young patients with PCOS had a high rate of AITD and this result was associated with high levels of fasting insulin and HOMA-IR. However, no correlation was established between autoantibody positivity and metabolic parameters such as BMI, insulin, and HOMA-IR.

In conclusion, we showed that insulin resistance was an important risk factor for increased TV and nodule formation. Patients with PCOS frequently have a thyroid disorder. Thyroid hormones are usually checked during the investigation of patients with PCOS; however, as a result of our study, serum thyroid autoantibodies and presence of thyroid nodule should also be investigated in these patients. The major limitation of our study is the determination of participants' glucose status only by measuring fasting glucose and HOMA index. However, the Clinical Practice Guidelines of the Endocrine Society recommend using an oral glucose tolerance test (OGTT) to screen for impaired glucose tolerance and type 2 diabetes (29). Further studies should be designed with large numbers of participants using OGTT.

Ethics Committee Approval: Ethics committee approval was received for this study from the ethics committee of Dişkapı Ylldirm Beyazt Training and Research Hospital.

Informed Consent: Written informed consent was obtained from patients who participated in this study.

Peer-review: Externally peer-reviewed.

Author Contributions: Concept - M.K., E.C.., M.S.A.; Design - M.K., S.H.; Supervision - M.K., E.C.., M.Ö.; Materials - M.K., 
S.H., E.T.; Data Collection and/or Processing - M.K., S.H., S..A.; Analysis and/or Interpretation - M.K., M.S.A., E.Ç.; Literature Review - M.K., Ş.A., İ.̈̈.; Writer - M.K., E.Ç.; Critical Review M.K., M.S.A.,E.Ç.

Conflict of Interest: No conflict of interest is declared by the authors.

Financial Disclosure: The authors declared that this study received no financial support.

\section{References}

1. Norman RJ, Dewailly D, Legro RS, Hickey TE. Polycystic ovary syndrome. Lancet 2007; 370: 685-97.

2. Amato MC, Vesco R, Vigneri E, Ciresi A, Giordano C. Hyperinsulinism and polycystic ovary syndrome (PCOS): role of insulin clearance. J Endocrinol Invest 2015; 38: 1319-26.

3. Ehrmann DA, Barnes RB, Rosenfield RL, Cavaghan MK, Imperial J. Prevalence of impaired glucose tolerance and diabetes in women with polycystic ovary syndrome. Diabetes Care 1999; 22: 141-6.

4. Coviello AD, Legro RS, Dunaif A. Adolescent girls with polycystic ovary syndrome have an increased risk of the metabolic syndrome associated with increasing and rogen levels independent of obesity and insulin resistance. J Clin Endocrinol Metab 2006; 91: 492-7.

5. Legro RS. Polycystic ovary syndrome and cardiovascular disease: a premature association. Endocr Rev 2003; 24: 302-12.

6. Hardiman P, Pillay OS, Atiomo W. Polycystic ovary syndrome and endometrial carcinoma. Lancet 2003; 361:1810-2.

7. McLeod DS, Cooper DS. The incidence and prevalence ofthyroid autoimmunity. Endocrine 2012; 42: 252-65.

8. Burguera B, Gharib H. Thyroid incidentelomas: Prevalence, diagnosis, significance, and management. Endocrinol Metab Clin North Am 2000; 29: 187-203.

9. Mortensen JD, Woolner LB, Bennet WA. Gross and microscopic findings in clinically normal thyroid glands. J Clin Endocrinol Metab 1955; 10: 1270-80.

10. Poppe K, Velkeniers B, Glinoer D. The role of thyroid autoimmunity in fertility and pregnancy. Nat Clin Pract Endocrinol Metab 2008; 4: 394-405.

11. Tomer Y, Huber A. The etiology of autoimmune thyroid disease: a story of genes and environment. J Autoimmun 2009; 32: 231-9.

12. Rezzonico J, Rezzonico M, Pusiol E, Pitoia F, Niepomniszcze $\mathrm{H}$. Introducing the thyroid gland as another victim of the insulin resistance syndrome. Thyroid 2008; 18: 461-4.

13. Rezzónico JN, Rezzónico M, Pusiol E, Pitoia F, Niepomniszcze $\mathrm{H}$. Increased prevalence of insulin resistance in patients with differentiated thyroid carcinoma. Metab Syndr Relat Disord 2009; 7: $375-80$.
14. Rotterdam ESHRE/ASRM-Sponsored PCOS consensus workshop group. Revised 2003 consensus on diagnostic criteria and longterm health risks related to polycystic ovary syndrome (PCOS). Hum Reprod 2004; 19: 41-7.

15. Matthews DR, Hosker JP, Rudenski AS, Naylor BA, Treacher DF, Turner RC. Homeostasis model assessment: insulin resistance and beta-cell function from fasting plasma glucose and insulin concentrations in man. Diabetologia 1985; 28: 412-9.

16. Duran C, Basaran M, Kutlu O, Kucukaydin Z, Bakdik S, Burnik FS, et al. Frequency of nodular goiter and autoimmune thyroid disease in patients with polycystic ovary syndrome. Endocrine 2015; 49: 464-9.

17. Sinha U, Sinharay K, Saha S, Longkumer TA, Baul SN, Pal SK. Thyroid disorders in polycystic ovarian syndrome subjects: A tertiary hospital based cross-sectional study from Eastern India. Indian J Endocrinol Metab 2013; 17: 304-9.

18. Ozdemir D, Cuhaci N, Balkan F, Usluogullari A, Ersoy R, Cakir B. Prevalence of thyroid pathologies in patients with polycystic ovary syndrome. Eur Cong Endocrinol 2011; 26: 92.

19. Rapoport B, Chazenbalk GD, Jaume JC, McLachlan SM. The thyrotropin (TSH) receptor: interaction with TSH and autoantibodies. Endocr Rev 1998; 19: 673-716.

20. Yasar HY, Ertuğrul O, Ertuğrul B, Ertuğrul D, Sahin M. Insulin Resistance in nodular thyroid disease. Endocr Res 2011; 36: 167-74.

21. Anil C, Kut A, Atesagaoglu B, Nar A, Bascil Tutuncu N, Gursoy A. Metformin Decreases Thyroid Volume and Nodule Size in Subjects with Insulin Resistance: A Preliminary Study. Med Princ Pract 2016; 25: 233-6.

22. Wellen KE, Hotamisligil GS. Inflammation, stress and diabetes. J Clin Invest 2005; 115: 1111-9.

23. Roith DL, Zick Y. Recent advances in our understanding of insulin action and insulin resistance. Diabetes Care 2001; 24: 588-97.

24. Vella V, Sciacca L, Pandini G, Mineo R, Squatrito S, Vigneri R, et al. The IGF-1 system in thyroid cancer: new concepts. Mol Pathol 2001; 54: $121-5$

25. Sahin M, Uçan B, Giniş Z, Topaloğlu O, Güngüneş A, Bozkurt NÇ, et al. Vitamin D3 levels and insulin resistance in papillary thyroid cancer patients. Med Oncol 2013; 30: 589.

26. Kachuei M, Jafari F, Kachuei A, Keshteli AH. Prevalence of autoimmune thyroiditis in patients with polycystic ovary syndrome. Arch Gynecol Obstet 2012; 285: 853-6.

27. Janssen OE, Mehlmauer N, Hahn S, Offner AH, Gärtner R. High prevalence of autoimmune thyroiditis in patients with polycystic ovary syndrome. Eur J Endocrinol 2004; 150: 363-9.

28. Calvar CE, Bengolea SV, Deutsch S, Hermes R, Ramos G, Loyato M. High frequency of thyroid abnormalities in polycystic ovary syndrome. Medicina (B Aires) 2015; 75: 213-7.

29. Legro RS, Arslanian SA, Ehrmann DA, Hoeger KM, Murad MH, Pasquali $\mathrm{R}$, et al. Diagnosis and treatment of polycystic ovary syndrome: an Endocrine Society clinical practice guideline. J Clin Endocrinol Metab 2013; 98: 4565-92. 